\title{
Mutant prominin 1 found in patients with macular degeneration disrupts photoreceptor disk morphogenesis in mice
}

\begin{abstract}
Zhenglin Yang, ${ }^{1,2}$ Yali Chen, ${ }^{1,3}$ Concepcion Lillo, ${ }^{4}$ Jeremy Chien, ${ }^{5}$ Zhengya Yu, ${ }^{1,3}$ Michel Michaelides, 6,7 Martin Klein, ${ }^{8}$ Kim A. Howes, ${ }^{1,3}$ Yang Li, ${ }^{1,3}$ Yuuki Kaminoh, 1,3 Haoyu Chen, 1,3 Chao Zhao,, 1,3 Yuhong Chen,, 1,3 Youssef Tawfik Al-Sheikh,1,3 Goutam Karan,, 1,3 Denis Corbeil, ${ }^{1}$ Pascal Escher, ${ }^{10}$ Shin Kamaya, ${ }^{1,3}$ Chunmei Li, ${ }^{1,3}$ Samantha Johnson, ${ }^{6}$ Jeanne M. Frederick, ${ }^{1}$ Yu Zhao, 1,3 Changguan Wang,,$^{1,3}$ D. Joshua Cameron, ${ }^{1,3}$ Wieland B. Huttner, ${ }^{11}$ Daniel F. Schorderet, ${ }^{10}$ Frances L. Munier, ${ }^{10}$ Anthony T. Moore, 6,7 David G. Birch, 8 Wolfgang Baehr, 1,12,13 David M. Hunt, ${ }^{6}$ David S. Williams, ${ }^{4,14}$ and Kang Zhang, ${ }^{1,3,13}$

1Department of Ophthalmology and Visual Science, Eccles Institute of Human Genetics, University of Utah, Salt Lake City, Utah, USA. ${ }^{2}$ Center for Human Molecular Biology and Genetics, Sichuan Academy of Medical Sciences, and Sichuan Provincial People's Hospital, Sichuan, People's Republic of China. ${ }^{3}$ Program in Human Molecular Biology and Genetics, Eccles Institute of Human Genetics, University of Utah, Salt Lake City, Utah, USA. ${ }^{4}$ Department of Pharmacology and Department of Neurosciences, UCSD School of Medicine, La Jolla, California, USA.

5Department of Laboratory Medicine and Experimental Pathology, Mayo Clinic College of Medicine, Rochester, Minnesota, USA.

${ }^{6}$ Institute of Ophthalmology, University College London, London, United Kingdom. ${ }^{7}$ Moorfields Eye Hospital, London, United Kingdom.

${ }^{8}$ Retina Foundation of the SW and Department of Ophthalmology, University of Texas Southwestern Medical Center, Dallas, Texas, USA.

${ }^{9}$ Tissue Engineering Laboratories, Biotec, Technical University of Dresden, Dresden, Germany. ${ }^{10}$ Institut de Recherche en Ophtalmologie, Sion, Switzerland. ${ }^{11}$ Max-Planck-Institute of Molecular Cell Biology and Genetics, Dresden, Germany. ${ }^{12}$ Department of Biology and ${ }^{13}$ Department of Neurobiology and Anatomy, University of Utah, Salt Lake City, Utah, USA. ${ }^{14}$ Jules Stein Eye Institute, UCLA School of Medicine, Los Angeles, California, USA.
\end{abstract}

\begin{abstract}
Familial macular degeneration is a clinically and genetically heterogeneous group of disorders characterized by progressive central vision loss. Here we show that an $\mathrm{R} 373 \mathrm{C}$ missense mutation in the prominin 1 gene (PROM1) causes 3 forms of autosomal-dominant macular degeneration. In transgenic mice expressing R373C mutant human PROM1, both mutant and endogenous PROM1 were found throughout the layers of the photoreceptors, rather than at the base of the photoreceptor outer segments, where PROM1 is normally localized. Moreover, the outer segment disk membranes were greatly overgrown and misoriented, indicating defective disk morphogenesis. Immunoprecipitation studies showed that PROM1 interacted with protocadherin 21 (PCDH21), a photoreceptor-specific cadherin, and with actin filaments, both of which play critical roles in disk membrane morphogenesis. Collectively, our results identify what we believe to be a novel complex involved in photoreceptor disk morphogenesis and indicate a possible role for PROM1 and PCDH21 in macular degeneration.
\end{abstract}

\section{Introduction}

Retinal photoreceptor cells are highly specialized. One compartment, organized as an outer segment (OS) in vertebrates, contains a stack of over 1,000 disks harboring rhodopsin and the phototransduction machinery. The entire OS is renewed by the continuous formation of new disks at its base and the shedding of older disks from its distal end (1). In humans and most other primates, the region comprising about $20^{\circ}$ in the center of the retina is known as the macula. Both rod and cone photoreceptor cells have regions of highest density in the macula, so that the macula provides high-acuity vision.

While genetically diverse and rare in prevalence, focus on familial macular degeneration has most often identified disease-causing gene mutations affecting photoreceptor and retinal pigment epithelial (RPE) cell function (2-7). Previously, we mapped 2 inherited autosomal-dominant macular degeneration phenotypes to an over-

Nonstandard abbreviations used: CNGCA1, rod cyclic nucleotide gated channel alpha subunit cook; ERG, electroretinography; IS, inner segment; LOD, logarithm of odds; MCDR2, bull's-eye macular dystrophy; ONL, outer nuclear layer; OS, outer segment; PCDH21, protocadherin 21; PROM1, prominin 1; RPE, retinal pigment epithelial/epithelium; STGD4, Stargardt disease 4.

Conflict of interest: The authors have declared that no conflict of interest exists. Citation for this article: J. Clin. Invest. 118:2908-2916 (2008). doi:10.1172/JCI35891. lapping region on chromosome 4p, a Stargardt-like macular dystrophy (Stargardt disease 4 [STGD4]; OMIM 603786; ref. 8) and bull'seye macular dystrophy (MCDR2; OMIM 608051; ref. 9). STGD4 is characterized by bilateral, symmetric, atrophic lesions in the macula and by the presence of yellow fundus flecks, whereas MCDR2 is characterized by bilateral annular RPE atrophy at the macula (9).

In the present study, genetic screening and mutation analysis of the 12 genes residing within this overlapping disease interval revealed an $\mathrm{R} 373 \mathrm{C}$ missense mutations in the prominin 1 gene (PROM1; GenBank accession no. AF027208, also known as CD133). PROM1 encodes a 5-transmembrane domain protein containing 2 large, highly glycosylated extracellular loops and a cytoplasmic tail (10). It is specifically associated with plasma membrane protrusions $(11,12)$, but its function and specific molecular interactions remain largely unknown. It is present in many tissues. In the retina, PROM1 is found at the base of the photoreceptor OSs, where the new disk membranes are formed, and has previously been linked to autosomal-recessive retinal degeneration $(13,14)$.

In order to study the cellular basis for macular degeneration caused by mutant PROM1, as well as the molecular function of PROM1 in photoreceptor cells, we generated transgenic mice carrying the R373C mutant PROM1 and studied the ensuing pathogenesis. Additionally, we carried out molecular interac- 
A

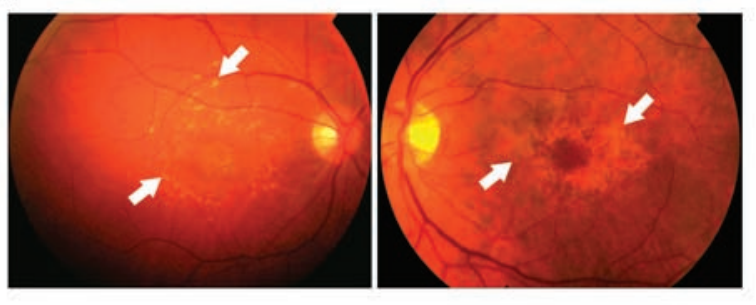

C
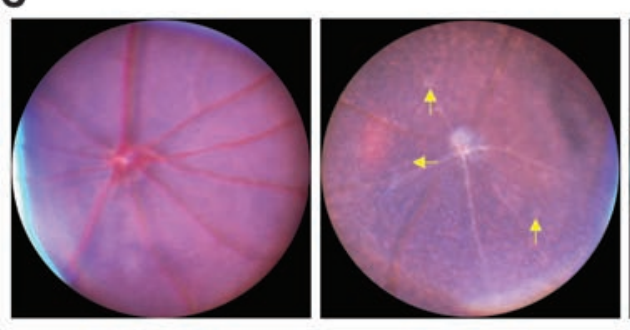

B
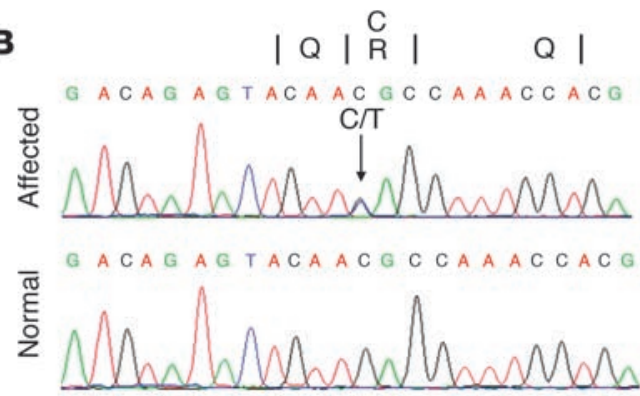

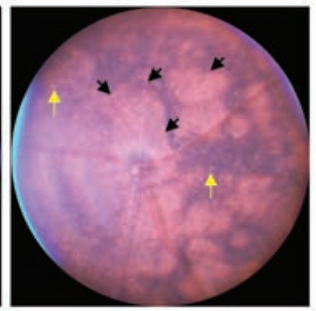

D

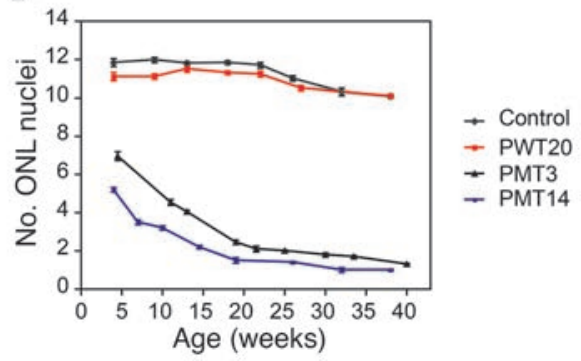

Figure 1

Retinal degeneration as a consequence of mutant PROM1. (A and B) Clinical features of autosomal-dominant macular dystrophy and segregation of a PROM1 mutation. (A) Left: Fundus photograph from a STGD4 patient with visual acuity of 20/200, showing an area of macular atrophy with surrounding yellow flecks (white arrows). Right: Fundus photograph from an MCDR2 patient with a visual acuity of 20/80, showing bull's-eye maculopathy (white arrows). (B) Sequencing traces demonstrating an $1117 \mathrm{C}>\mathrm{T}$ transition in exon 10 giving rise to the missense R373C substitution in both families. (C) Fundus photographs from representative 12-month-old PTW20 (left), 4-month-old PMT14 (middle), and 13-month-old PMT14 (right) mice. Yellow arrows denote yellow deposits and small lesions scattered throughout the central fundus. Black arrows denote large coalescing deposits and atrophic lesions in the fundus. (D) Progressive photoreceptor loss, as determined by the number of nuclei in the ONL, in PMT3 and PMT14 mice compared with normal C57BL/6 (control) and PWT20 mice. Photoreceptor cell nuclei were counted in transgenic mice between 4 and 40 weeks of age. Error bars, which in most cases are too small to be visible, represent \pm SEM. The rate of photoreceptor cell loss was greater in the PMT14 line, which expressed higher levels of mutant PROM1 than did PMT3 mice (see Figure 3).

tion studies with PROM1 to investigate its potential role in disk membrane morphogenesis.

\section{Results}

Identification of the PROM1 mutation in STGD4, MCDR2, and conerod dystrophy pedigrees. Previously, we identified family pedigrees showing STGD4, clinically characterized by macular dystrophy and yellow fundus flecks (8), and an autosomal-dominant MCDR2, characterized by RPE atrophy (9) (Figure 1A and Supplemental Figure 1, A and B; supplemental material available online with this article; doi:10.1172/JCI35891DS1). In this study we narrowed the disease interval to a $12-\mathrm{cM}$ region between markers D4S1582 and GATA1582G03. We screened 12 candidate genes and identified a R373C missense mutation in PROM1 in both phenotypes (Figure 1B). We then identified the same R373C mutation in a third pedigree with an autosomaldominant cone-rod dystrophy characterized by both cone and rod photoreceptor degeneration (Supplemental Figure 1C). Comparison of disease haplotypes revealed that affected members in the STGD4, MCDR2, and cone-rod dystrophy families had distinct disease haplotypes, indicating that the PROM1 mutation in each family arose independently (Supplemental Figure 1). The logarithm of odds (LOD) score in each family was $7.7,2.1$, and 4.4, respectively, and the combined LOD score of the 3 families was 14.2 at $\theta$ of 0 with marker D4S403. The mutation segregated with affected individuals in each family (LOD score 5.8, 1.7, 4.3; combined LOD, 11.8 at $\theta$ of 0 ), and was absent in 400 normal matched controls. PROM1 was expressed in both cone and rod photoreceptors (Supplemental Figure 2).

Generation of transgenic mice expressing WT PROM1 and the R373C buman PROM1 mutation. To gain insight into the molecular mechanism of retinal degeneration caused by mutant PROM1, we generated transgenic mice carrying the R373C mutant human PROM1 mutation under the control of the 4.4-kb rhodopsin promoter (15). Expression of the human PROM1 transgene in the retina was verified by Western blotting (Supplemental Figure 3A). As a control for overexpression of PROM1 in mice, we also generated transgenic mice expressing human WT PROM1. We characterized 2 human WT (PWT20 and PWT31) and 2 mutant (PMT3 and PMT14) PROM1 transgenic mouse lines. By Western blot we found that the relative expression levels of WT (PWT20 and PWT31) and mutant (PMT14 and PMT3) human PROM1 transgenes were 1-, 2-, 8-, and 2-fold, respectively, that of endogenous mouse Prom1 expression (Supplemental Figure 3B).

WT and mutant PROM1 transgenic mouse phenotypes. Fundus images and light microscopy of retinal sections from transgenic mice expressing mutant PROM1 revealed progressive retinal abnormalities visible as subretinal deposits and photoreceptor atrophy (Figure 1C and Supplemental Figure 4). Compared with PWT20 mice, PMT14 mice showed fundus changes characteristic of changes seen in humans with Stargardt disease. Yellow deposits and small lesions (Figure 1C, yellow arrows) were scattered throughout the 

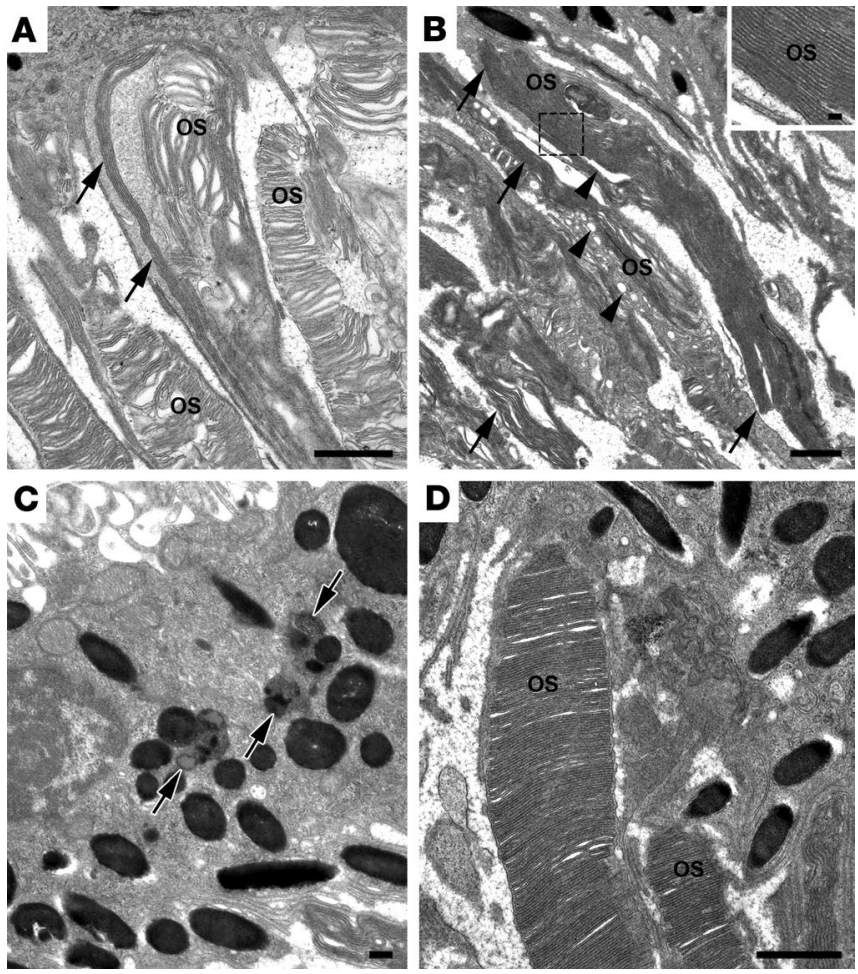

Figure 2

Electron microscopy of $P R O M 1$ transgenic mouse rod photoreceptors. (A and B) Overgrown and abnormally oriented disk membranes (arrows) in PMT3 retinas were visible at 16 days (A) and 4 months (B) of age. Arrowheads indicate vesiculated membrane. Higher-magnification view of the boxed region in B is shown in the inset. (C) Electron micrograph showing abnormalities in the RPE of PMT3 mice at 4 months of age. Accumulation of lipofuscin-like material (arrows) was evident. (D) Electron micrograph of a WT retina, showing normal OS disk membranes and adjacent RPE. Scale bars: $1 \mu \mathrm{m}$ (A, B, and D); 300 nm (C); 30 nm (B, inset).

central fundus in 4-month-old PMT14 mice. By 13 months of age, large coalescing deposits and atrophic lesions (Figure 1C, black arrows) covered much of the fundus. With fluorescein angiography, the atrophic lesions showed hyperfluorescence at the earlier phase and hypofluorescence in the late phase, indicating a window defect and RPE atrophy (data not shown).

Mutant PROM1 transgenic mice exhibited progressive photoreceptor degeneration, measured by loss of photoreceptor nuclei in the outer nuclear layer (ONL; Figure 1D and Supplemental Figure 4). The rate of photoreceptor loss correlated with expression levels in the mutant PROM1 mouse lines, with 50\% of photoreceptors lost from the regions located $200-300 \mu \mathrm{m}$ dorsal and ventral to the optic nerve head at 4 weeks in PMT14 mice and 7 weeks in PMT3 mice (Figure 1D). In contrast, higher levels of WT PROM1 transgene expression did not cause retinal abnormalities or degeneration (Figure 1D and Supplemental Figures 4 and 5).

Electron microscopy revealed that most of the rod photoreceptor OSs of mutant PROM1 retinas were disorganized. By 16 days after birth, during photoreceptor development, overgrown disk membranes were evident in PMT3 retinas (Figure 2A, arrows). By 4 months of age, the remaining photoreceptors contained either vesiculated OS membranes (Figure $2 \mathrm{~B}$, arrowheads) or stacks of disk membranes that were of excessive size and aligned perpendicular to normal disk orientation (Figure $2 \mathrm{~B}$, arrows and inset). The OSs of a WT retina are shown in Figure 2D. In the RPE, there were abnormal lipofuscin-like deposits (Figure 2C, arrows), indicating that this cell type was also compromised. Such deposits were not present in 4-month-old control (i.e., WT or PWT20) mice.

Electroretinography (ERG) recordings were performed to assess retinal function in PM14 mice (Supplemental Figure 5A). The rod and cone ERG b-waves were both significantly reduced relative to normal C57BL/ 6 controls ( $t$ test, $2.9 ; P<0.01$ ) and PWT20 mice ( $t$ test, $2.1 ; P<0.05)$ at 3 months of age, indicating that both rod and cone photoreceptor cells are affected by mutant PROM1. $\mathrm{B}$-wave responses were not detectable in the PM14 line at 9 months of age. Correspondingly, maximum high-intensity rod a-wave responses, which are proportional to the total number of functioning photoreceptor disks, were reduced by an average of $50 \%$ at 3 months and were not detectable at 9 months of age (Supplemental Figure 5B).

WT and mutant PROM1 localization was examined by immunohistochemistry in transgenic and control mouse retinas. PROM1 was previously observed by immunoelectron microscopy to be localized to the nascent disk membranes at the base of rod OSs in control retinas (13). Our immunofluorescence images were consistent with this finding; label was restricted to a narrow region that corresponded to base of the photoreceptor OSs (Figure 3, A-C). In PWT20 mice, PROM1 showed a similar localization pattern (Figure 3, D-F). Conversely, in PMT3 and PMT14 mice, mutant PROM1 was found most strongly in the myoid region of photoreceptors, a site containing predominantly ER, and throughout photoreceptors extending from OSs to the synaptic termini (Figure 3, G and J). The endogenous mouse Prom 1 in these mutant retinas appeared to show mislocalization throughout the photoreceptor layers (Figure 3, H and K). Nevertheless, PROM1 immunofluorescence should be interpreted with caution, because Western blot analysis showed low levels of PROM1 expression in PMT3 and PMT14 retinas (Supplemental Figure 3C).

Protocadherin 21 localization in PROM1 transgenic mice. Protocadherin 21 (PCDH21; GenBank accession no. AB053448), previously shown to also localize to the nascent disks at the base of the OSs, is thought to play a critical role in disk membrane morphogenesis (16). Based on its location similar to that of PROM1, we immunolabeled PCDH21 in WT and mutant PROM1 transgenic mice. In PWT20 mice, PCDH21 colocalized with WT PROM1 at the base of OSs (Figure 3, M-O). In mutant PROM1 retinas, PCDH21 was mislocalized in a pattern similar to that of PROM1 throughout photoreceptors (Figure 3, P-R). In contrast, immunolocalization of retinal OS membrane protein 1 (ROM1; found in the rims of the mature disk membranes; refs. 17, 18), rod cyclic nucleotide gated channel alpha subunit cook (CNGCA1; an OS marker; ref. 19), and $\mathrm{Na}^{+} / \mathrm{K}^{+}$-ATPase (an inner segment [IS] marker; ref. 20) proteins were not disturbed in mutant PROM1 mice (Supplemental Figure 6), indicating that mutant PROM1 does not have a generalized effect on intracellular vectorial or compartmental transport.

Based on these results, we also examined immunolocalization

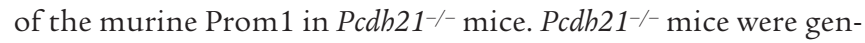
erated previously and were shown to form disorganized OSs and undergo photoreceptor degeneration (16). Using the mousespecific Prom 1 antibody, we found WT Prom 1 mislocalized throughout OSs in the absence of Pcdh21 (Figure 3, S-U). Similar to the mutant PROM1 transgenic mice, the Pcdh21 $1^{-/-}$mice 

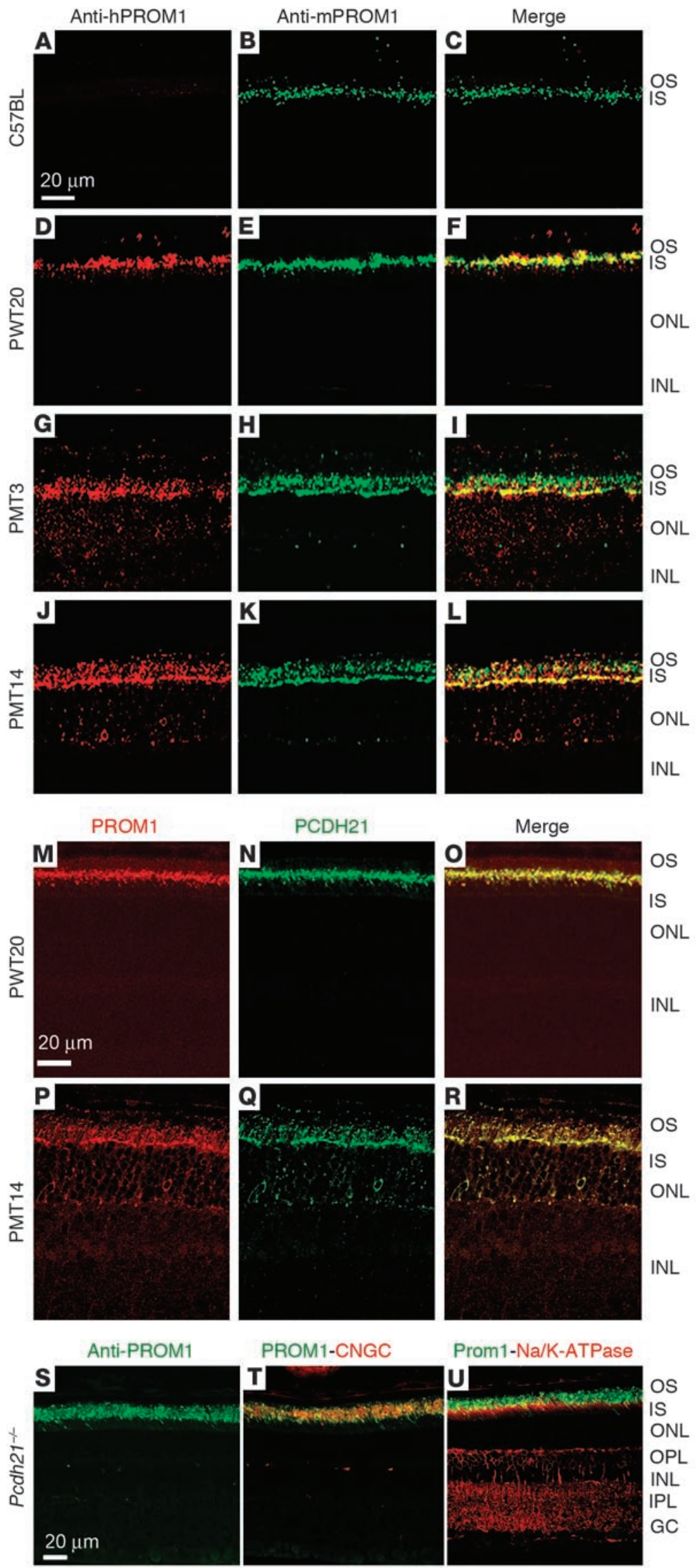

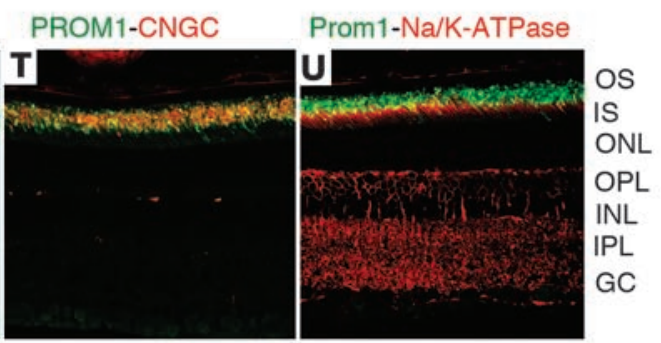

Figure 3

Immunohistochemical localization of human PROM1 and endogenous mouse PROM1 in PROM1 transgenic retinas at 1 month of age. (A-L) Localization of transgenic human PROM1 (red) and native mouse PROM1 (green). (A-C) C57BL/6 mice showed endogenous mouse PROM1 signal alone at the base of the OSs. (D-F) In PWT20 mice, human PROM1 and endogenous mouse PROM1 were normally localized to the base of the OSs. (G-L) In PMT3 and PMT14 retinas, mutant PROM1 was mislocalized throughout the photoreceptors from the ONL to the OSs, whereas endogenous mouse PROM1 signal was more restricted to the ISs and OSs. Both endogenous mouse PROM1 and mutant PROM1 showed strong immunolabeling in the myoid region of the ISs. (M-R) PROM1 (red) and PCDH21 (green) protein immunolocalization in WT and mutant $P R O M 1$ transgenic retinas. (M-O) In 1-month-old PWT20 mice, PCDH21 and PROM1 colocalized at the base of photoreceptor OSs. (P-R) In PMT14 mice, PCDH21 was mislocalized, as was PROM1, to the ONL. (S-U) WT PROM1 immunolocalization in Pcdh21 $1^{-1}$ mice. WT PROM1 immunolabeling (S) was evident throughout OSs. PROM1 colocalized with the OS marker CNGCA1 (T) and not the IS marker, Na/K-ATPase (U). Note that CNGCA1 and $\mathrm{Na} / \mathrm{K}-\mathrm{ATP}$ ase signals were correctly located within OS and IS compartments, similar to the normal immunolabeling pattern in the R373C mutant PROM1 transgenic mice. INL, inner nuclear layer; OPL, outer plexiform layer; IPL, inner plexiform layer; GC, ganglion cells. Scale bars: $20 \mu \mathrm{m}$.

did not show perturbations in CNGCA1 (Figure $3 \mathrm{~T}$ ) or $\mathrm{Na}^{+} / \mathrm{K}^{+}$-ATPase (Figure $3 \mathrm{U}$ ) locations in the OS or IS photoreceptor regions, respectively. These results suggest that the interaction of these proteins is required for normal Prom 1 localization to the base of OSs and that this interaction plays a role in normal disk morphogenesis.

Physical interaction of PROM1 and PCDH21. Migration of WT PROM1 on SDS gel reflected the predominance of the glycosylated form $(\sim 114 \mathrm{kDa})$ when HEK293 cells were transfected with PROM1 constructs (Figure 4A, lane 1). Infrequently, an additional faster-moving PROM1 polypeptide $(\sim 105$ $\mathrm{kDa}$ ) was detected (Figure 4A, lane 3).

Because PCDH21 was mislocalized with mutant PROM1 in transgenic mice, we carried out coimmunoprecipitation studies to determine whether PROM1 and PCDH21 directly interact. HEK293 cells were cotransfected with either WT or mutant PROM1 and a PCDH21-Myc fusion construct. Interestingly, we found that WT and mutant PROM1 coimmunoprecipitated with PCDH21 (Figure 4A, lanes 2 and 4). Reciprocally, PCDH21 coimmunoprecipitated with the human PROM1 antibody (Figure 4B, lanes 2 and 3 ). To confirm their in vivo interaction, the PCDH21 antibody was used in immunoprecipitation reactions with WT and mutant PROM1 transgenic mouse retinal lysates. Western blot analysis showed the interaction of PCDH21 protein with WT and mutant PROM1 in vivo (Figure 4C). 
A

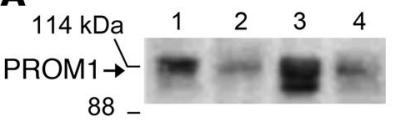

B

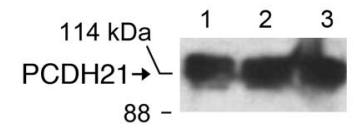

C

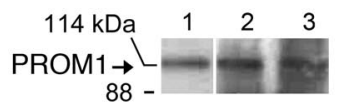

D

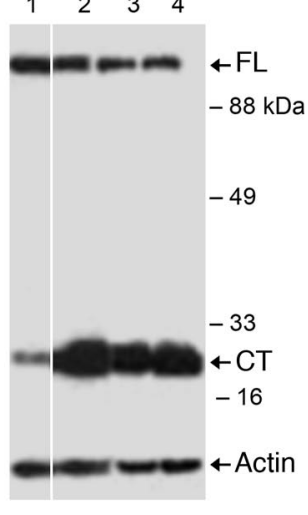

Figure 4

Coimmunoprecipitation of PROM1 and PCDH21 and proteolytic cleavage of PCDH21. (A) HEK293 cells were cotransfected with PCDH21 and either WT or mutant PROM1. Immunoprecipitation was performed with anti-PCDH21, followed by anti-PROM1 immunodetection on a Western blot. Immunolabeling of WT (lane 1) and mutant (lane 3) PROM1 in the transfected cell lysates (anti-PROM1 Western blot) served as positive controls. Also shown is immunolabeling of WT (lane 2) and mutant (lane 4) PROM1 in the PCDH21 immunoprecipitates. (B) Reciprocal immunoprecipitation with PROM1 antibody after HEK293 cells were cotransfected with PCDH21-Myc and WT (lane 2) or mutant (lane 3) PROM1. Lane 1 shows WT PCDH21-Myc-transfected cell lysate (anti-Myc Western blot) as a positive control. (C) Immunoprecipitation of retinal lysates. Lysates from WT (lane 2) and mutant (lane 3) PROM1 transgenic mice were immunoprecipitated with anti-PCDH21, followed by anti-human PROM1 detection on a Western blot. Lane 1 is a PROM1 protein positive control, showing PROM1 immunolabeling of retinal lysate. (A-C) Results of negative control experiments performed using nonspecific IgG were negative. (D) Proteolytic processing of PCDH21 in PROM1 transgenic mice. Full-length $(\mathrm{FL})$ and proteolytic C-terminal (CT) PCDH21 fragments were compared among PMT3 (lane 1), C57BL/6 (lane 2), PWT31 (lane 3), and PWT20 (lane 4) mice. Total retinal protein lysate $(20 \mu \mathrm{g})$ was probed with the PCDH21 C terminus antibody; $\beta$-actin was used as a loading control. PMT3 retinas showed substantially more full-length PCDH21 and less cleaved fragment than did PWT20, PWT31 and C57BL/6 retinas. Lanes were run on the same gel but were noncontiguous.

PCDH21 undergoes proteolytic processing releasing the ectodomain and retaining the transmembrane and intracellular domains during disk morphogenesis (21). We observed an increase in levels of unprocessed, full-length PCDH21 $(\sim 125 \mathrm{kDa})$ and decreased levels of the cleaved fragment $(\sim 25 \mathrm{kDa})$ in cell lysates from mutant PROM1 mouse retinas compared with WT PROM1 transgenic and C57BL/6 retinas (Figure 4D). These results suggested that the R373C mutation may also affect proteolytic processing of PCDH21 in mutant PROM1 mice.

Physical interaction of PROM1 with actin. The overgrown and misoriented disk membranes in R373C PROM1 transgenic mice (Figure $2, A$ and B) are strikingly similar to those previously observed in retinas treated with cytochalasin $\mathrm{D}$ in order to depolymerize actin filaments $(22,23)$. Hence, we explored whether there is an interaction between PROM1 and actin filaments and whether any interaction would be affected by the R373C mutation. Cells transfected with WT PROM1 showed protrusions and membrane processes, and some of the PROM1 colocalized with actin, especially in the protrusions (Supplemental Figure 7). In contrast, cells transfected with mutant PROM1 did not possess protrusions, and the mutant

PROM1 did not colocalize with actin (Supplemental Figure 7). We found a physical interaction between WT PROM1 and actin by immunoprecipitation in transfected cells (Figure 5) and in transgenic retinas (Supplemental Figure 8), and this interaction was markedly reduced by the R373C mutation (Figure 5). Furthermore, polymerization of actin filaments in photoreceptors was affected in mutant PROM1 transgenic mice compared with that in WT transgenic mice (Supplemental Figure 9). Taken together, these results indicate that PROM1 can interact with actin filaments and that this interaction is decreased by the $\mathrm{R} 373 \mathrm{C}$ mutation.

\section{Discussion}

We report that a missense mutation in PROM1 causes 3 forms of autosomal-dominant retinal dystrophy. In transgenic mice expressing mutant PROM1, the mutant protein was mislocalized, and defects were evident in both rod and cone photoreceptor cells. ERG responses were reduced, and photoreceptor cell loss occurred at a rate that correlated with the expression level of the mutant PROM1. Most notably, the mutation in PROM1 interfered with its interaction with PCDH21 and actin, and prior to degeneration, the disks of the photoreceptor OSs were aberrant. Both these observations support a role for PROM1 in the morphogenesis of new disk membranes.

PROM1 and retinal degeneration. Mutations in the PROM1 gene have been previously linked to pedigrees with autosomal-recessive retinitis pigmentosa $(13,14)$, where they mainly affect rod photoreceptors and peripheral vision. These mutations were frameshift mutations, resulting in premature stop codons and truncation of the encoded protein, most likely representing null mutations. In transfection studies, the truncated protein was found to be degraded quickly; a minor amount was detected in the endoplasmic reticulum and it was absent from the cell surface, so that the degeneration in these patients appeared to have resulted from loss of PROM1 function (13). In contrast, the R373C mutation results in a stable mutant protein, and, as we show here, causes dominant macular degeneration that mainly affects cone photoreceptors and central vision. The R373C mutant PROM1 was mislocalized and caused the mislocalization of WT Prom 1 as well as that of at least one other critical protein, PCDH21.

Interaction with PCDH21. Rattner et al. $(16,21)$ described a photoreceptor cadherin, PCDH21; like PROM1, PCDH21 localizes to the nascent disks of rod and cone OSs. Moreover, like the PROM1 R373C mutant mice, $P c d h 21^{-/-}$mice exhibit disorganized photoreceptor membrane disks in OSs with corresponding photoreceptor degeneration (16). The present results support a functional interaction between PROM1 and PCDH21. PROM1 and PCDH21 were colocalized at the base of OSs, and PROM1 was coimmunoprecipitated with full-length PCDH21. Further evidence of this interaction was obtained upon analysis of molecular abnormalities in R373C PROM1 transgenic mice. PCDH21 showed an overlapping mislocalized immunolabeling pattern with the R373C mutant protein in photoreceptors. With immunoblots, we found a marked reduction in cleaved PCDH21 levels in R373C mutant retinal lysates. Given that loss of PCDH21 results in retinal degeneration (16), the dominant effect of the R373C PROM1 mutation on retinal degeneration could stem from impairment of PCDH21 function.

Because PROM1 and PCDH21 are both present on the nascent disks, their interaction appears likely to be within the same membrane or between adjacent membranes of 2 nascent disks. In the latter case, they may play a role in maintaining the spacing between 

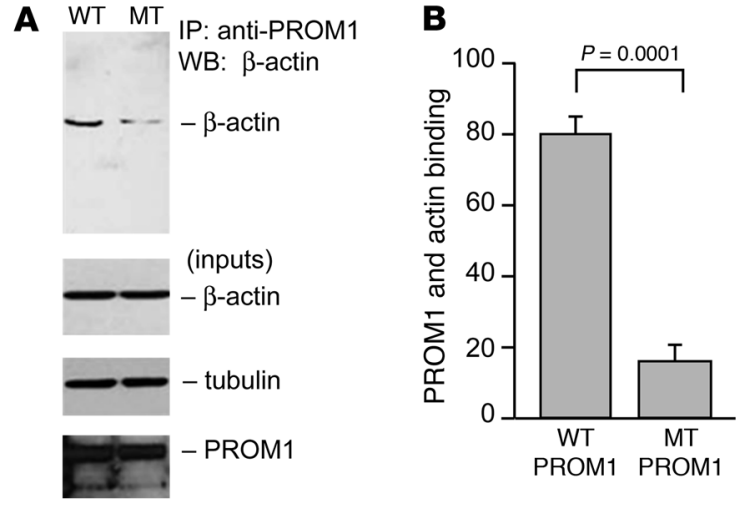

\section{Figure 5}

Coimmunoprecipitation of PROM1 and $\beta$-actin. (A) HEK293 cells were transfected with either WT or mutant (MT) PROM1. Whole cell lysates with equal amounts of input WT or mutant PROM1 were immunoprecipitated with PROM1 polyclonal antibody, followed by anti$\beta$-actin immunodetection on Western blots (WB). (B) Quantification of PROM1 and $\beta$-actin interaction by densitometry. Values (mean \pm SD) denote the amount of $\beta$-actin, immunoprecipitated with PROM1 antibodies from cells, transfected with WT or mutant PROM1. Each experiment was done in triplicate, and 3 independent transfection experiments were performed for each PROM1 construct. Significance was examined using independent sample $t$ test.

neighboring disk membranes. Interestingly, Drosophila prominin is present on the tips of the rhabdomeral microvilli; together with the protein spacemaker, it is responsible for keeping the rhabdomeres apart, resulting in the formation of an open, rather than a closed, rhabdom (24).

Disk membrane morphogenesis. Steinberg et al. (25) proposed that the morphogenesis of disk membranes at the base of rod and cone OSs occurs by 2 membrane growth phases: the evagination of the ciliary plasma membrane followed by the formation of the disk rim. Although this model has gained experimental support over the years, Chuang et al. (26) recently revived another model, proposed by Obata and Usukura (27), in which vesicles fuse to form nascent disks that are assembled entirely within the cell's plasma membrane. One of the weaknesses of this fusion model is the assumption that the nascent disks are closed and do not differ from the mature disks except in size. A variety of reports have shown that the nascent disks of rod OSs have several distinct characteristics. Unlike the more mature disks, the nascent disks can be labeled by Lucifer yellow administered to the extracellular space (28); they contain specific proteins, such as PCHD21 (16, 21) and PROM1; and they specifically exclude some mature disk proteins, such as the rim protein peripherin/rds (29). Moreover, cone photoreceptor OSs, which in many species do not contain any closed disks, provide a clear demonstration of the presence of open disks (29-32). The membrane fusion presented in support of a fusion model (26) likely arises from a preservation artifact, along the lines described by Townes-Anderson (33).

In addition to its specific localization in nascent disks, previous data on PROM1 and our present results provide additional support to the evagination/rim growth model. PROM1 is best characterized as a component of membrane domains that are protruding and have high curvature (10-12). Recently, PROM1 was detected at the tips of primary cilia on the neuroepithelium of the neocortex
(34). Membrane was observed to bud off from these cilia (34), in a manner reminiscent of that from photoreceptor cilia in $r d s$ mice (35), which lack peripherin/rds (2) and are thus unable to form the rims of the disk membranes.

In the present study, we observed overgrown disk membranes, oriented abnormally along the OS plane, in R373C PROM1 transgenic mice (Figure 2, A and B). A strikingly similar phenotype has previously been described following the depolymerization of actin filaments with cytochalasin D in eyecup cultures and in retinas in vivo $(22,23)$. In these studies, observations of especially the cone disk membranes provided strong evidence that the overgrowth was caused by continued membrane evagination (23). The overgrown disks incorporated newly synthesized protein, and their formation appeared to result from a failure in the initiation of new disk membranes - the initial protrusion of the ciliary plasma membrane was inhibited, so that the new membrane was channeled into nascent disks that were already growing (23). In addition to actin filaments, it seems likely that PROM1 is also required for the normal, regular initiation of new disks. This suggestion is consistent with the general association of PROM1 on membrane protrusions (refs. 10-12 and Supplemental Figure 7), including those from primary cilia (34), and our present finding that PROM1 bound actin (Figure 5). Mice lacking RPGRIP, which interacts with the retinitis pigmentosa GTPase regulator (RPGR) in the photoreceptor connecting cilium, also produce overgrown disk membranes, and it has been suggested that RPGRIP may regulate actin cytoskeletal dynamics (36).

In summary, our results identify what we believe to be a novel molecular complex involved in photoreceptor disk membrane morphogenesis and support the model whereby new disk membranes form by an evagination of the ciliary plasma membrane followed by rim growth.

\section{Methods}

Patients. Approval from the institutional review boards of the University of Utah, Moorfields Eye Hospital, and Institut de Recherche en Ophtalmologie was obtained for this study, and informed consent was obtained from all patients. Participating in the study were 27 individuals at risk for inheriting STGD4 in the first kindred, from the United States, a black family; 9 individuals at risk for inheriting MCDR2 in the second kindred, from England, a family of mixed European descent; and 15 individuals at risk for inheriting cone-rod dystrophy in the third kindred, from Switzerland.

Genetic linkage and mutation screening. DNA was extracted from blood samples, and genetic linkage was assessed using microsatellite markers D4S1582, D4S2906, D4S403, D4S2362, GGAT18G02, D4S1525, D4S2946, D4S2633, D4S2926, and GATA158G03 (tightly linked to the STGD4 and MCDR2 loci) using previously established methods (6). We performed 2-point linkage analysis using the FASTLINK (37) version of MLINK from the LINKAGE Program Package (38). An autosomal-dominant mode of inheritance with full penetrance was used for LOD score computation. Disease allele frequency was set at 0.0001 (39). PROM1 mutation screening was performed by denaturing HPLC (dHPLC) analysis followed by direct sequencing of PCR-amplified DNA fragments for all 23 PROM1 exons using previously established methods (6). PCR primers were designed to include flanking intronic sequences of each exon according to published protocols (6). Amplified products were purified using the QIAquik Gel Extraction Kit (Qiagen) and sequenced with forward and reverse primers by the Taq Dyedeoxy Terminator Cycle Sequencing Kit (Beckman-Coulter) according to the manufacturer's instructions. dHPLC analysis was used according to the manufacturer's instructions (Transgenomics Inc.) 
to screen for changes in PROM1 sequence and to determine the presence or absence of the mutation in the controls.

Generation of expression constructs. Forward and reverse primers (5'CCGCTCGAGCGTTGCTAGCTATGGCCCTC-3' and 5'-ATAGTTTAGCGGCCGCATTCTTATTCAATGTTGTGATGGGCTTGTC-3', respectively) containing $X h o l$ and Notl restriction sites were designed for amplification of WT human PROM1 cDNA. Following Xhol and Notl restriction digest, the amplified cDNA was cloned into the Xhol and Notl sites of the pcDNA3.1(-) vector (Invitrogen). The $1117 \mathrm{C}>\mathrm{T}(R 373 \mathrm{C})$ mutation was introduced into the WT PROM1 pcDNA3 construct by PCR-based site-directed mutagenesis. The recombinant plasmids were purified using a Qiagen plasmid isolation kit (Qiagen). Mouse PCDH21 expression constructs were generously provided by A. Rattner and J. Nathans (Johns Hopkins University School of Medicine, Baltimore, Maryland, USA; ref. 21). All constructs were verified by restriction digestion and DNA sequencing.

Generation of PROM1 transgenic mice. WT or mutant human PROM1 cDNAs, generated as described above, were subcloned into the pRHO4.4 plasmid, containing the 4.4-kb human rbodopsin promoter - directing expression to rod and cone photoreceptors (15) - and a bovine poly A site. All constructs were verified by restriction enzyme digestion and direct DNA sequencing. Inserts were isolated by Notl and Kpnl digestion, microinjected into C57BL/ 6 mouse embryos, and implanted into pseudopregnant foster female mice. Founder mice were identified by PCR. Human PROM1 primers (forward, 5'-CCGCTCGAGCGTTGCTAGCTATGGCCCTC-3'; reverse, 5'-CGGGATCCCGCTATCAATGTTGTGATGGGC-3') were used for PCR analysis of genomic DNA extracted from mouse tail biopsies. WT and mutant transgenic lines were established and propagated in the C57BL/6 mouse strain. All procedures were approved by the IACUC of the University of Utah and carried out according to NIH guidelines.

PROM1 expression in transgenic mice. After removal of the lens, retinas from 1-month-old mice were manually separated from the RPE and lysed in 150 $\mathrm{mM} \mathrm{NaCl} ; 50$ mM Tris, pH 7.5; 1 mM EDTA; 1\% Triton X-100; 0.5\% SDS; and protease inhibitor mixture (Roche Applied Science). Protein from each retinal lysate $(10 \mu \mathrm{g})$ was separated by SDS-PAGE, transferred to PVDF filters, and probed with either human PROM1- or mouse Prom1-specific antibodies, followed by incubation with HRP-conjugated secondary antibodies and standard ECL detection.

Fundus photography and fluorescein angiography. Fundus photographs and fluorescein angiographs for C57BL/6 and WT and mutant PROM1 transgenic mice were recorded at 6 and 9 months of age using a Kowa RC- 2 handheld fundus camera (Kowa Genesis). The eyes were dilated with scopolamine ( $0.25 \%$; Isopto Hyoscine; Alcon) $1 \mathrm{~h}$ prior to photography. Fluorescein angiographs were recorded with negative black and white film after intraperitoneal injection of $0.2 \mathrm{ml} 25 \%$ sodium fluorescein diluted $1: 1$ with sterile PBS.

Histology and light microscopy. Mice were maintained in a continuous 12-h light/12-h dark cycle and sacrificed 8-12 h after the onset of the light phase. Anesthetized mice were perfused with $0.1 \mathrm{M} \mathrm{PBS}$ and then with $2.5 \%$ glutaraldehyde in $0.1 \mathrm{M}$ PBS ( $\mathrm{pH} 7.4$ ) by intracardiac injection. The superior sclera of each eye was marked for orientation. Eyecups were processed for embedding in Epon. Sections of $0.5 \mu \mathrm{m}$, oriented along the dorsoventral axis of the retina and containing the optic nerve head, were used for measuring photoreceptor ONL width. Photoreceptor nuclear counts were measured within 200-300 $\mu \mathrm{m}$ dorsal and ventral regions flanking the optic nerve head. Five separate counts per side (total 10 counts) were averaged, and ONL width was expressed as average number of nuclei.

Dark-adapted ERG. Electroretinograms were obtained from 42 mice between the ages of 3 and 15 months: 19 PMT14, 8 PWT20, and 15 C57BL/6. Several mice were tested at more than one age. Mice were dilated with $0.25 \%$ scopolamine, dark-adapted overnight, and prepared for recording under a dim red light while the dilation was reinforced with scopol- amine. Mice were anesthetized with intraperitoneal injection of 0.008 $\mathrm{ml} / \mathrm{g}$ of a mixture of ketamine $(20 \%)$, xylazine $(0.5 \%)$, and sodium chloride solution (79.5\%). The corneal electrode was a Burian-Allen mouse electrode referenced to a needle electrode in the scalp. A second needle electrode in the tail served as ground. Mice were placed between 2 heating pads to stabilize body temperature, which was continuously monitored with a digital probe. The eye was numbed with proparacaine hydrochloride (Alcaine; Alcon) and Refresh Celluvisc (Allergan Inc.) was added to the contact lens electrode to protect the eye. Signals were amplified $10,000 \times$ and filtered (8-pole Butterworth $60-\mathrm{Hz}$ notch filter) to remove line noise before averaging $(n=20-200)$ by computer. A ganzfeld dome and the incorporated Grass photostimulator, similar to systems used in human testing, was used to produce flashes comparable to the International Society for the Clinical Electrophysiology of Vision standard (40). Rod a-waves were elicited by a high-intensity Xenon flash (Novatron). The rod phototransduction model was fit to the leading edges of a-waves generated in response to high-intensity stimuli, and the dashed curves were the best fitting curves (41). Cone $\mathrm{b}$-wave responses were obtained in the presence of a rod-saturating background (3.2 log photopic trolands).

Electron microscopy. Eyes from WT and mutant PROM1 transgenic mice were fixed in $2 \%$ paraformaldehyde plus $2 \%$ glutaraldehyde in $0.1 \mathrm{M}$ cacodylate buffer, $\mathrm{pH} 7.4$, and embedded in Epon 812 resin. Ultrathin sections were mounted on copper grids and stained with uranyl acetate and lead citrate and examined with a Philips electron microscope (model 208).

Immunohistochemistry. Antibodies used in these studies included rabbit polyclonal anti-human PROM1 (42); rat monoclonal anti-mouse Prom1 (11); rabbit polyclonal anti-mouse PCDH21 (diluted 1:500; gift from A. Rattner and J. Nathans; ref. 16); mouse monoclonal anti-mouse CNGCA1 (diluted 1:100; gift from R. Molday, University of British Columbia, Vancouver, British Columbia, Canada; ref. 19); mouse monoclonal anti-mouse ROM1 (diluted 1:100; gift from R. Molday; ref. 43); and rabbit polyclonal anti-mouse $\mathrm{Na}^{+} / \mathrm{K}^{+}$-ATPase (diluted 1:100; obtained from the Developmental Studies Hybridoma Bank, University of Iowa).

Cryostat sections $(12 \mu \mathrm{m})$ were washed with $1 \times$ PBS, blocked in immunohistochemical buffer $(0.2 \%$ Triton X-100 in PBS) containing $10 \%$ goat serum at room temperature for $1 \mathrm{~h}$. Sections were incubated in primary antibody overnight at $4{ }^{\circ} \mathrm{C}$. After washing, sections were incubated in FITCconjugated goat anti-rabbit or Texas Red-conjugated goat anti-mouse secondary antibodies (diluted 1:100; Invitrogen) at room temperature for $1 \mathrm{~h}$. Immunofluorescence was examined, and images were captured on a Zeiss 510 confocal microscope.

Cell transfection and immunoprecipitation reactions of PROM1 and PCDH21. Cultured cells were propagated in DMEM (Gibco; Invitrogen) supplemented with $10 \%$ FBS (Gibco; Invitrogen), $100 \mathrm{U} / \mathrm{ml}$ penicillin, and 100 $\mu \mathrm{g} / \mathrm{ml}$ streptomycin. Cells were transfected with recombinant human WT or mutant PROM1 and mouse recombinant PCDH21 (containing a C-terminal Myc epitope tag) with FuGENE 6 Transfection Reagent (Roche Applied Science) according to the manufacturer's recommended protocol. At $36 \mathrm{~h}$ after transfection, cells were lysed in $150 \mathrm{mM} \mathrm{NaCl} ; 50 \mathrm{mM}$ Tris, $\mathrm{pH} 7.5 ; 1$ mM EDTA; $1 \%$ Triton X-100; 0.5\% SDS; and a protease inhibitor mixture (Roche Applied Science). Cell lysates were centrifuged at $1,600 \mathrm{~g}$ for $5 \mathrm{~min}$ at $4^{\circ} \mathrm{C}$ to remove nuclei and insoluble material. The anti-PROM1 antibody was used to immunoprecipitate PROM1-binding proteins. The immunoprecipitated proteins were separated by SDS-PAGE, transferred to PVDF filters, and probed with the Myc antibody on Western blot. The reciprocal experiment was performed using the PCDH21 antibody for immunoprecipitations followed by Western blot with anti-PROM1. For retinal coimmunoprecipitation studies, retinas were isolated and pooled from $10 \mathrm{WT}$ or mutant PROM1 transgenic mice, and protein was extracted as described above. 
Western blots of PROM1 and PCDH21 in transgenic mouse retinas. Dissected retinas were briefly homogenized in buffer containing $150 \mathrm{mM} \mathrm{NaCl} ; 50$ mM Tris, pH 7.5; 1 mM EDTA; $1 \%$ Triton X-100; 0.5\% SDS; and complete protease inhibitor mixture (Roche Applied Science). Following a 20-min incubation on ice, lysate was cleared of nuclei and insoluble material by centrifugation at $1,600 \mathrm{~g}$ for $5 \mathrm{~min}$ at $4^{\circ} \mathrm{C}$. Soluble proteins were separated by SDS-PAGE, after which Western blot analysis was performed. The polyclonal PCDH21 C-terminal antibody was used to detect fulllength PCDH21 and the C-terminal proteolytic fragment in C57BL/6 and PROM1 transgenic mice. A polyclonal antibody specific for human PROM1 without cross-reactivity to the mouse Prom 1 was used to detect WT and mutant PROM1 transgene expression. An anti-mouse $\beta$-actin antibody (Sigma-Aldrich) was used as a loading control. For visualization, blots were probed with a HRP-conjugated anti-rabbit or anti-mouse secondary antibody (diluted 1:4,000; GE Healthcare) and developed with an ECL kit according to the manufacturers' protocol (GE Healthcare).

Immunoprecipitation of PROM1 and actin. HEK293 cells transfected with WT or mutant PROM1 were lysed in the wash buffer (50 mM Tris, pH 7.5; 150 $\mathrm{mM} \mathrm{NaCl} ; 1 \% \mathrm{NP}-40$; and $0.5 \%$ sodium deoxycholate) supplemented with complete protease inhibitors (Roche Applied Science), centrifuged at 10,000 g for 5 min to obtain postnuclear supernatant, and immunoprecipitated using affinity-purified polyclonal PROM1 antibodies. Immunocomplexes were precipitated by Protein A-Agarose (Pierce Biotechnologies Inc.) and washed 4 times with wash buffer. Immunoprecipitated protein samples were eluted with Laemmli buffer with $100 \mathrm{mM}$ DTT, resolved by $10 \%$ SDS-PAGE, and immunoblotted with $\beta$-actin monoclonal antibodies (Sigma-Aldrich).

Dissected retinas from 1-month-old PWT20 mice were homogenized in buffer containing $150 \mathrm{mM} \mathrm{NaCl} ; 50 \mathrm{mM}$ Tris, pH 7.5; 1 mM EDTA; 1\% Triton X-100; 0.5\% SDS; and complete protease inhibitor mixture (Roche Applied Science). Lysate was centrifuged at $10,000 \mathrm{~g}$ for $5 \mathrm{~min}$ to obtain postnuclear supernatant and then immunoprecipitated using affinity-purified polyclonal PROM1 antibody. Immunocomplexes were precipitated by Protein A-Agarose, resolved by $10 \%$ SDS-PAGE, and immunoblotted with a $\beta$-actin monoclonal antibody.

Immunostaining and fluorescence microscopy of PROM1 and actin. HEK293 cells were transfected with WT or mutant PROM1. Immunostaining was performed essentially as described previously (44). Slides were incubated for $1 \mathrm{~h}$ at room temperature in anti-PROM1 (diluted 1:500) or rhodamineconjugated phalloidin for F-actin (diluted 1:2,000). Laser-scanning confocal microscopy was performed on a Zeiss LSM510 microscope with krypton-argon and helium-neon lasers.

Cryosections of PWT20 and PM3 retinas were labeled with FITC-conjugated phalloidin (Invitrogen) to visualize actin filaments and with tubulin antibody. Immunofluorescence was examined, and images were captured on a Zeiss 510 confocal microscope.

1. Young, R.W. 1967. The renewal of photoreceptor cell outer segments. J. Cell Biol. 33:61-72.

2. Travis, G.H., Brennan, M.B., Danielson, P.E., Kozak, C.A., and Sutcliffe, J.G. 1989. Identification of a photoreceptor-specific mRNA encoded by the gene responsible for retinal degeneration slow (rds). Nature. 338:70-73.

3. Connell, G., et al. 1991. Photoreceptor peripherin is the normal product of the gene responsible for retinal degeneration in the rds mouse. Proc. Natl. Acad. Sci. U. S. A. 88:723-726.

4. Allikmets, R., et al. 1997. Mutation of the Stargardt disease gene (ABCR) in age-related macular degeneration. Science. 277:1805-1807.

5. Weng, J., et al. 1999. Insights into the function of Rim protein in photoreceptors and etiology of Stargardt's disease from the phenotype in abcr knockout mice. Cell. 98:13-23.

6. Zhang, K., et al. 2001. A 5-bp deletion in ELOVL4

Statistics. Significance of differences was determined using SPSS software. Comparisons were made by 2 -tailed Student's $t$ test. A $P$ value less than 0.05 was considered significant.

\section{Acknowledgments}

We thank patients and their families for their participation; R. Molday, A. Rattner, and J. Nathans for antibody reagents; A. Rattner and J. Nathans for pcdh21/prCAD knockout mice; and Kirsten Locke for mouse fundus photography. K. Zhang was supported by NIH grants R01EY14428, R01EY14448, P30EY014800, and GCRC M01-RR00064; a Foundation Fighting Blindness Center grant; Research to Prevent Blindness; the Ruth and Milton Steinbach Fund; Ronald McDonald House Charities; the Macular Vision Research Foundation; a Burroughs Wellcome Fund Clinical Scientist Award in Translational Research; a VA Merit Award; the Grant Ritter Fund; and the Val and Edith Green Foundation. D.M. Hunt and A.T. Moore were supported by the British Retinitis Pigmentosa Society and the Guide Dogs for the Blind Association. D.S. Williams was supported by NIH grants R01EY07042 and P30EY12598. D.G. Birch was supported by NIH grant EY05235 and a Foundation Fighting Blindness Center grant. W. Baehr and J.M. Frederick were supported by NIH grant RO1 EY08123 and a Foundation Fighting Blindness Center grant. D. Corbeil and W.B. Huttner were supported by Deutsche Forschungsgemeinschaft grants SFB/TR13-04 B1, SFB 655 A2 (to W.B. Huttner), and A13 (to D. Corbeil). Z. Yang was supported by the American Health Assistance Foundation. D.S. Williams is a Jules and Doris Stein RPB Professor, and K. Zhang is an RPB Lew Wasserman Merit Award Scholar.

Received for publication April 9, 2008, and accepted in revised form June 4, 2008.

Address correspondence to: Zhenglin Yang or Kang Zhang, Department of Ophthalmology, Shiley Eye Center, UCSD, 9415 Campus Drive, San Diego, California 92037, USA. Phone: (858) 246-0040; Fax: (858) 534-8293; E-mail: zliny@yahoo.com (Z. Yang), kangzhang@ucsd.edu (K. Zhang). Or to: David S. Williams, Doris Stein Eye Research Center, 200 Stein Plaza, Los Angeles, California 90095-7002, USA. Phone: (310) 825-9546; Fax: (310) 825-9546; E-mail: dswilliams@ucla.edu.

Zhenglin Yang's and Kang Zhang's present address is: Department of Ophthalmology, Shiley Eye Center, UCSD, San Diego, California, USA.

is associated with two related forms of autosomal dominant macular dystrophy. Nat. Genet. 27:89-93.

7. Karan, G., et al. 2005. Lipofuscin accumulation, abnormal electrophysiology, and photoreceptor degeneration in mutant ELOVL4 transgenic mice: A model for macular degeneration. Proc. Natl. Acad. Sci.U. S. A. 102:4164-4169.

8. Kniazeva, M.F., et al. 1999. Clinical and genetic studies of an autosomal dominant cone-rod dystrophy with features of Stargardt disease. Ophthalmic Genet. 20:71-81.

9. Michaelides, M., et al. 2003. An autosomal dominant bull's-eye macular dystrophy (MCDR2) that maps to the short arm of chromosome 4. Invest. Ophthalmol. Vis. Sci. 44:1657-1662.

10. Corbeil, D., Roper, K., Fargeas, C.A., Joester, A., and Huttner, W.B. 2001. Prominin: a story of cholesterol, plasma membrane protrusions and human pathology. Traffic. 2:82-91.
11. Weigmann, A., Corbeil, D., Hellwig, A., and Huttner, W.B. 1997. Prominin, a novel microvillispecific polytopic membrane protein of the apical surface of epithelial cells, is targeted to plasmalemmal protrusions of non-epithelial cells. Proc. Natl. Acad. Sci. U. S. A. 94:12425-12430.

12. Corbeil, D., et al. 2000. The human AC133 hematopoietic stem cell antigen is also expressed in epithelial cells and targeted to plasma membrane protrusions. J. Biol. Chem. 275:5512-5520.

13. Maw, M.A., et al. 2000. A frameshift mutation in prominin (mouse)-like 1 causes human retinal degeneration. Hum. Mol. Genet. 9:27-34.

14. Zhang, Q., et al. 2007. Severe retinitis pigmentosa mapped to 4 p 15 and associated with a novel mutation in the PROM1 gene. Hum. Genet. 122:293-299.

15. Woodford, B.J., Chen, J., and Simon, M.I. 1994. Expression of rhodopsin promoter transgene product in both rods and cones. Exp. Eye Res. 
58:631-635.

16. Rattner, A., et al. 2001. A photoreceptor-specific cadherin is essential for the structural integrity of the outer segment and for photoreceptor survival. Neuron. 32:775-786.

17. Bascom, R.A., Liu, L., Heckenlively, J.R., Stone, E.M., and McInnes, R.R. 1995. Mutation analysis of the ROM1 gene in retinitis pigmentosa. Hum. Mol. Genet. 4:1895-1902.

18. Clarke, G., et al. 2000. Rom-1 is required for rod photoreceptor viability and the regulation of disk morphogenesis. Nat. Genet. 25:67-73.

19. Cook, N.J., Molday, L.L., Reid, D., Kaupp, U.B., and Molday, R.S. 1989. The cGMP-gated channel of bovine rod photoreceptors is localized exclusively in the plasma membrane. J. Biol. Chem. 264:6996-6999.

20. Stahl, W.L., and Baskin, D.G. 1984. Immunocytochemical localization of $\mathrm{Na}^{+}, \mathrm{K}+$ adenosine triphosphatase in the rat retina. J. Histochem. Cytochem. 32:248-250

21. Rattner, A., Chen, J., and Nathans, J. 2004. Proteolytic shedding of the extracellular domain of photoreceptor cadherin. Implications for outer segment assembly. J. Biol. Chem. 279:42202-42210.

22. Vaughan, D.K., and Fisher, S.K. 1989. Cytochalasin D disrupts outer segment disc morphogenesis in situ in rabbit retina. Invest. Ophthalmol. Vis. Sci. 30:339-342.

23. Williams, D.S., Linberg, K.A., Vaughan, D.K., Fariss, R.N., and Fisher, S.K. 1988. Disruption of microfilament organization and deregulation of disk membrane morphogenesis by cytochalasin D in rod and cone photoreceptors. J. Comp. Neurol. 272:161-176

24. Zelhof, A.C., Hardy, R.W., Becker, A., and Zuker, C.S. 2006. Transforming the architecture of compound eyes. Nature. 443:696-699.
25. Steinberg, R.H., Fisher, S.K., and Anderson, D.H. 1980. Disc morphogenesis in vertebrate photoreceptors. J. Comp. Neurol. 190:501-508.

26. Chuang, J.Z., Zhao, Y., and Sung, C.H. 2007. SARAregulated vesicular targeting underlies formation of the light-sensing organelle in mammalian rods. Cell. 130:535-547.

27. Obata, S., and Usukura, J. 1992. Morphogenesis of the photoreceptor outer segment during postnatal development in the mouse (BALB/c) retina. Cell Tissue Res. 269:39-48.

28. Matsumoto, B., and Besharse, J.C. 1985. Light and temperature modulated staining of the rod outer segment distal tips with Lucifer yellow. Invest. Oph thalmol. Vis. Sci. 26:628-635.

29. Arikawa, K., Molday, L.L., Molday, R.S., and Williams, D.S. 1992. Localization of peripherin/rds in the disk membranes of cone and rod photoreceptors: relationship to disk membrane morphogenesis and retinal degeneration. J. Cell Biol. 116:659-667.

30. Nilsson, S.E. 1964. Receptor cell outer segment development and ultrastructure of the disk membranes in the retina of the tadpole (Rana Pipiens). J. Ultrastruct. Res. 11:581-602.

31. Anderson, D.H., Fisher, S.K., and Steinberg, R.H. 1978. Mammalian cones: disc shedding, phagocytosis, and renewal. Invest. Ophthalmol. Vis. Sci. 17:117-133.

32. Carter-Dawson, L.D., and LaVail, M.M. 1979. Rods and cones in the mouse retina. I. Structural analysis using light and electron microscopy. J. Comp. Neurol. 188:245-262.

33. Townes-Anderson, E. 1995. Intersegmental fusion in vertebrate rod photoreceptors. Rod cell structure revisited. Invest. Ophthalmol. Vis. Sci. 36:1918-1933.

34. Dubreuil, V., et al. 2007. Midbody and primary cilium of neural progenitors release extracellular membrane particles enriched in the stem cell mark- er prominin-1. J. Cell Biol. 176:483-495.

35. Usukura, J., and Bok, D. 1987. Changes in the localization and content of opsin during retinal development in the rds mutant mouse: immunocytochemistry and immunoassay. Exp. Eye Res. 45:501-515.

36. Zhao, Y., et al. 2003. The retinitis pigmentosa GTPase regulator (RPGR)- interacting protein: subserving RPGR function and participating in disk morphogenesis. Proc. Natl. Acad. Sci. U. S. A 100:3965-3970

37. Cottingham, R.W., Idury, R.M., and Schaffer, A.A. 1993. Faster sequential genetic linkage computations. Am. J. Hum. Genet. 53:252-263.

38. Lathrop, G.M., and Lalouel, J.M. 1984. Easy calculations of lod scores and genetic risks on small computers. Am. J. Hum. Genet. 36:460-465.

39. Lathrop, G.M., Lalouel, J.M., Julier, C., and Ott, J. 1984. Strategies for multilocus linkage analysis in humans. Proc. Natl. Acad. Sci. U. S. A. 81:3443-3446.

40. Marmor, M.F. 1989. An international standard for electroretinography. Doc. Ophthalmol. 73:299-302.

41. Hood, D.C and Birch, D.G. 1993. Light adaptation of human rod receptors: the leading edge of the human a-wave and models of rod receptor activity. Vision Res. 33:1605-1618.

42. Florek, M., et al. 2005. Prominin-1/CD133, a neural and hematopoietic stem cell marker, is expressed in adult human differentiated cells and certain types of kidney cancer. Cell Tissue Res. 319:15-26.

43. Bascom, R.A., et al. 1992. Localization of the photoreceptor gene ROM1 to human chromosome 11 and mouse chromosome 19: sublocalization to human 11q13 between PGA and PYGM. Am. J. Hum. Genet. 51:1028-1035.

44. Lingle, W.L., and Salisbury, J.L. 2001. Methods for the analysis of centrosome reproduction in cancer cells. Methods Cell Biol. 67:325-336. 